\title{
Ausgezeichnete europäische Allergieforschung
}

$M$

it dem Ziel der Forschungsförderung auf dem Gebiet der Dermatologie und ihrer Grenzgebiete hat die Europäische Stiftung für Allergieforschung (ECARF) den mit 5.000 Euro dotierten „ADF/ECARF Award for European Research“verliehen. Der Preis ging zu gleichen Teilen an die beiden besten Beiträge, die während der Jahrestagung der Arbeitsgemeinschaft Dermatologische Forschung (ADF) vom 23. bis 25. März 2006 in Aachen vorgestellt wurden.

Die genauen Mechanismen der Toleranzentwicklung gegenüber Allergenen sind immer noch wenig bekannt. Für ihre Untersuchung des „Niedrigzo- nentoleranzmodells“, demzufolge die wiederholte Gabe geringer Allergenmengen eine Sensibilisierung gegenüber Kontaktallergenen verhindern kann, wurde das Forscherteam um Priv.-Doz. Dr. Kerstin Steinbrink, UniversitätsHautklinik Mainz, der „ADF/ECARF Award for European Allergy Research“ verliehen.

Die zweite Preishälfte ging an die Arbeitsgruppe um Prof. Dr. Thomas Werfel von der Hautlinik Linden der Medizinischen Hochschule Hannover. Sein Team hat erforscht, welche Bedeutung dem Zytokin IL-13 für die Entzündung bei der atopischen Dermatitis zukommt.

\section{Forschungspreis für Kinderpneumologin}

- pisodisch auftretende Rasselgeräu- sche beim Atmen findet man bei Säuglingen und Kleinkindern relativ häufig. Meist werden sie als Asthma interpretiert und entsprechend mit Bronchodilatatoren und inhalativen Glukokortikoiden behandelt. Einem beträchtlichen Teil der Kinder wird man aber mit dieser rein empirischen Vorgehensweise nicht gerecht, wie Dr. Sejal Saglani vom Royal Brompton Hospital in London, Großbritannien, in einer retrospektiven Studie zeigen konnte. Die

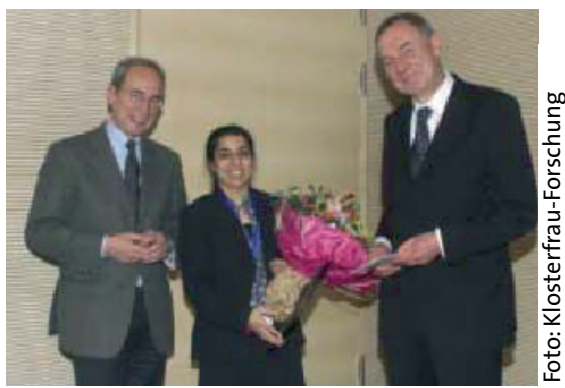

Der Vorsitzende der Expertenjury Prof. Dr. Dietrich Reinhardt (links), Dr. von Haunersches Kinderspital der Universität München, und Dr. Harald Greve (rechts), Leitung Wissenschaft und Medizin von Klosterfrau, mit der Gewinnerin des Klosterfrau Forschungspreises 2006, Dr. Sejal Saglani
Pneumologin unterzog dazu 47 Kinder mit episodischem Giemen einem zum Teil invasiven Untersuchungsprogramm, das unter anderem 24-h-pHMessungen im Ösophagus, Bronchoskopien mit broncho-alveolärer Lavage und endobronchiale Biopsien umfasste. Als häufigster Befund fand sich bei zwei Drittel der untersuchten Kinder ein gastroösophagealer Reflux. Die Bronchoskopie erwies sich bei den Kindern als praktikabel und sicher und lieferte wertvolle Zusatzbefunde. Die Ergebnisse machen deutlich, dass es sich bei Kindern mit rezidivierendem Giemen und Versagen der Asthmatherapie lohnt, auch mit invasiveren Untersuchungsmethoden nach weiteren Befunden zu fahnden.

Für ihre Arbeit wurde Dr. Saglani anlässlich der 28. Jahrestagung der Deutschen Gesellschaft für pädiatrische Pneumologie im März in Frankfurt/Main mit dem „Klosterfrau Forschungspreis 2006“ ausgezeichnet. Der internationale Preis ist mit 30.000 Euro dotiert und honoriert jedes Jahr besonders herausragende Forschungsarbeiten, die sich mit der Bekämpfung des kindlichen Asthma bronchiale beschäftigen. 